\title{
Hierarchical Surrogate-Assisted Evolutionary Multi-Scenario Airfoil Shape Optimization
}

\author{
Handing Wang*, John Doherty ${ }^{\dagger}$, and Yaochu Jin* \\ *Department of Computer Science, University of Surrey, Guildford, GU2 7XH, UK \\ Email: \{handing.wang, yaochu.jin\}@ surrey.ac.uk \\ ${ }^{\dagger}$ Department of Mechanical Engineering Sciences, University of Surrey, Guildford, GU2 7XH, UK \\ Email: john.doherty@ surrey.ac.uk
}

\begin{abstract}
For multi-scenario airfoil shape optimization problems, an evaluation of a single airfoil is based on its full-scenario drag landscape. To obtain the full-scenario drag landscape, a large number of computational fluid dynamic simulations for different operating conditions must be conducted. Since a single computational fluid dynamic simulation is often time-consuming, evaluations for multi-scenario airfoil shape optimization will be computationally highly intensive. Although surrogate-assisted evolutionary algorithms have been widely applied to expensive optimization problems, existing surrogate-assisted evolutionary algorithms cannot be directly applied to multi-scenario airfoil shape optimization. Instead of using surrogate models to directly approximate the multi-scenario evaluations, we employ a hierarchical surrogate model consisting of a K-nearest neighbors classifier and a Kriging model to approximate the full-scenario drag landscape for each candidate design during the optimization. Then, the fitness of the candidate design is evaluated based on the approximated drag landscape to reduce the computational cost. The proposed hierarchical surrogate model is embedded in the covariance matrix adaptation evolution strategy and applied to the RAE2822 airfoil design problem. Our experimental results show that the proposed algorithm is able to obtain an airfoil design with limited computational cost that perform well in different operating conditions.
\end{abstract}

\section{INTRODUCTION}

Due to the uncertainty of the operating scenario (or environmental condition), it is common that more than one fixed operating scenario should be considered for engineering design problems [1]. The performance of a design varies in different operating scenarios. A design is meaningless if it is optimal in one single scenario but performs badly in other scenarios. Taking a car design as an example, various scenarios (road conditions) may occur during driving and result in different performance, thus a well-designed car is expected to achieve relatively satisfactory performance in possible scenarios.

In the community of evolutionary computation (EC), a few studies for multi-scenario optimization have been reported, focusing on formulating a multi-scenario optimization problem. The existing formulation of multi-scenario optimization problems can be classified into two categories [2]: aggregation and multi-criterion methods. Although it is straightforward to aggregate scenarios as the objective function [3], assigning the weights to different scenarios is very hard when the uncertainty in the scenario space cannot be quantitatively estimated [4]. Therefore, formulating a multi-scenario optimization problem as a multi-objective optimization problem
[5], [6], [7], where each scenario is considered as an objective, is another formulation. When the number of the considered scenarios is more than three, the problem becomes a manyobjective optimization problem, whose optimum is a large set of solutions [8]. It is hard to choose one optimal solution from the set without any preference of scenarios.

The scenario space can be multi-dimensional and continuous. A large number of discrete scenarios need to be specified and are involved in every single evaluation during the optimization process, which increases the computational cost. The situation becomes even worse if the evaluation for each scenario is driven by expensive simulations like computational fluid dynamic (CFD) simulations which take hours for a single run. Additionally, both existing formulations of multi-scenario optimization are generic, neither taking any domain knowledge into account. They cannot be directly applied to specified engineering design problems.

The operating scenarios of an airfoil varies with different lifts and speed (termed as mach coefficient). Those different scenarios result in different aerodynamic performance. The airfoil shape design problem is a typical multi-scenario optimization problem. Therefore, the challenges of multi-scenario airfoil shape optimization problems are two-fold. Firstly, since its $2 \mathrm{D}$ continuous scenario space has a wide range, a large number of scenarios are considered for a full-scenario evaluation. Secondly, one full-scenario drag landscape is needed for a single evaluation, which costs too many expensive CFD simulations, thus the optimization process becomes even more expensive [9]. To reduce the high computational cost, cheap surrogate models [10] are used to approximate the full-scenario drag landscape of a given airfoil. With the approximated full-scenario drag landscape, the airfoil can be further evaluated for the optimization process. In this work, we choose covariance matrix adaptation evolution strategy (CMA-ES) [11] to search the optimal airfoil based on the approximated drag landscape, due to its fast convergence speed.

In the remainder of this paper, we firstly introduce multiscenario airfoil design in Section II, where the disadvantages of single-scenario airfoil design, the challenges of multiscenario airfoil design, and the problem formulation are discussed. Then, in Section III, the proposed algorithm, a hierarchical surrogate-assisted multi-scenario evolution strategy, is 
described in detail. To validate its performance, we apply the proposed algorithm to the RAE2822 airfoil design problem in Section IV. Section V concludes the paper.

\section{Multi-Scenario Airfoil Design}

Generally, an aircraft operates in various scenarios from take-off to landing, which results in very different aerodynamic performance. It is very often that the performance of a found optimal airfoil is evaluated in the whole scenario space. Since single-scenario evaluations based on CFD simulations are already expensive for designers to process optimization algorithms, full-scenario evaluations are even more expensive. To the best of our knowledge, no research on airfoil design using full-scenario evaluations has been reported.

\section{A. Drawback of Single-Scenario Airfoil Design}

Single-scenario optimization methods have widely been used for airfoil design, where the optimizer searches for solutions according to the performance for a scenario (a fixed lift $C_{L}$ and a fixed velocity $M$ ). Although the resulting designs perform optimally for the given scenario, their performance in other scenarios might seriously degrade.

Take the RAE5225 airfoil design problem as an example. The operating conditions of the airfoil can be defined by $C_{L} \in[0.3,0.75] \times M \in[0.5,0.76]$. We use a single-scenario method and multi-scenario optimization method, respectively, to search for optimal designs. The single-scenario optimization method minimizes the drag in one scenario $\left(C_{L}=0.7, M=\right.$ $0.725)$, and the multi-scenario optimization method minimizes the average drag of five different scenarios $\left(C_{L}=0.65\right.$, $M=0.725 ; C_{L}=0.7, M=0.72 ; C_{L}=0.7, M=0.725$; $M=0.7, C_{L}=0.73$; and $C_{L}=0.75, M=0.725$.) The fullscenario drag landscape $C_{D w}$ of the baseline design, the design obtained the single-scebnario optimization method, and one obtained by the optimizer considering five scenarios are shown in the top, middel and bottom panels of Fig. 1, respectively. Compared with the baseline design, the geometry obtained by the single-scenario optimization method has a small drag in the scenario of $C_{L}=0.7, M=0.725$, but the drag in the adjacent scenarios rapidly increases, resulting in poor performance in the whole scenario space [9]. From Fig. 1, we can also see that the full-scenario drag landscape of the design obtained by the multi-scenario optimization method is better than that of the single-scenario optimization method.

The above illustrative example demonstrates that multiscenario optimization can overcome the disadvantage of single-scenario optimization. The more scenarios are considered during the optimization, the better the full-scenario performance will be. Since five scenarios, which is only a small part of the entire scenario space, are considered in the above example, its full-scenario performance remains unsatisfactory. Note, however, that the computational cost for quality evaluations will dramatically increase when the number of the considered scenarios increases. Therefore, the main obstacle that prevents multi-scenario airfoil shape optimization
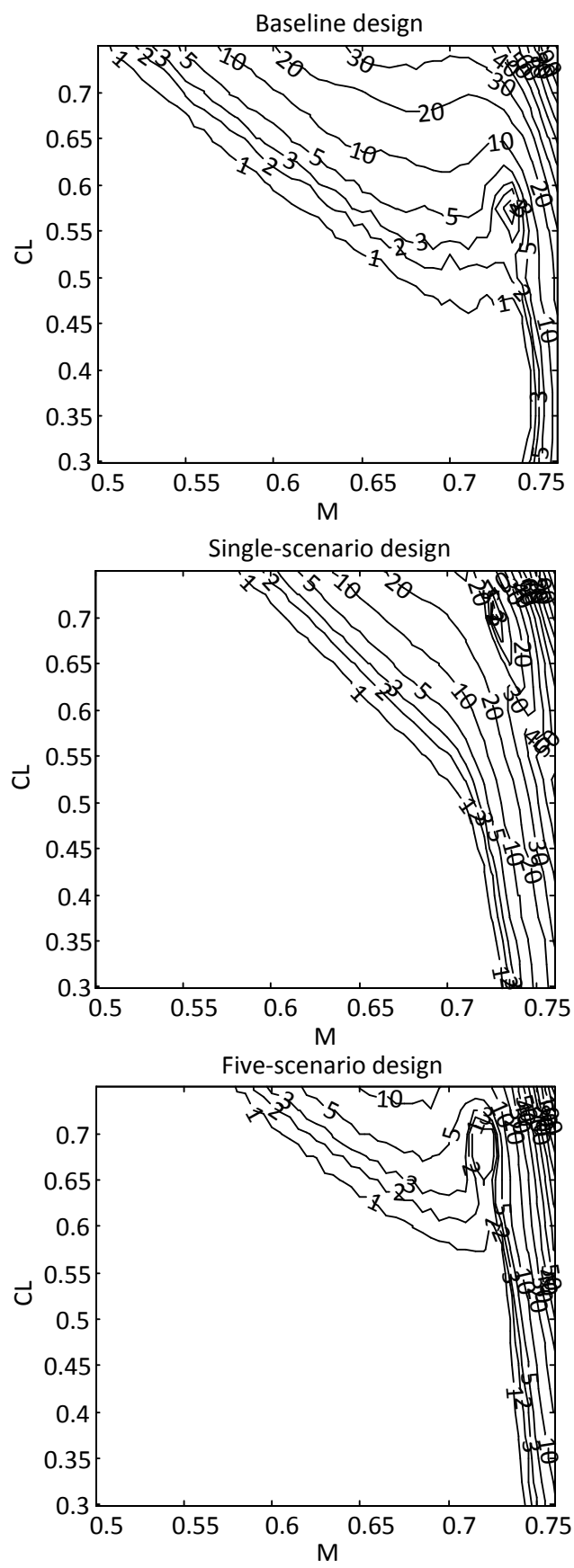

Fig. 1. Wave drag coefficient distribution of an RAE5225 airfoil designed by single- and multi-scenario optimization methods.

from being employed in expensive optimization such as airfoil design is the prohibitive computational cost.

\section{B. Problem Formulation for Multi-Scenario Airfoil Design}

Ideally, airfoil design should be formulated as a multiscenario optimization problem to take all main operating conditions into account. In the following, we discuss the problem formulation of multi-scenario airfoil design, including geometry parameterization (coding of decision variables) and definition of the objective function. 


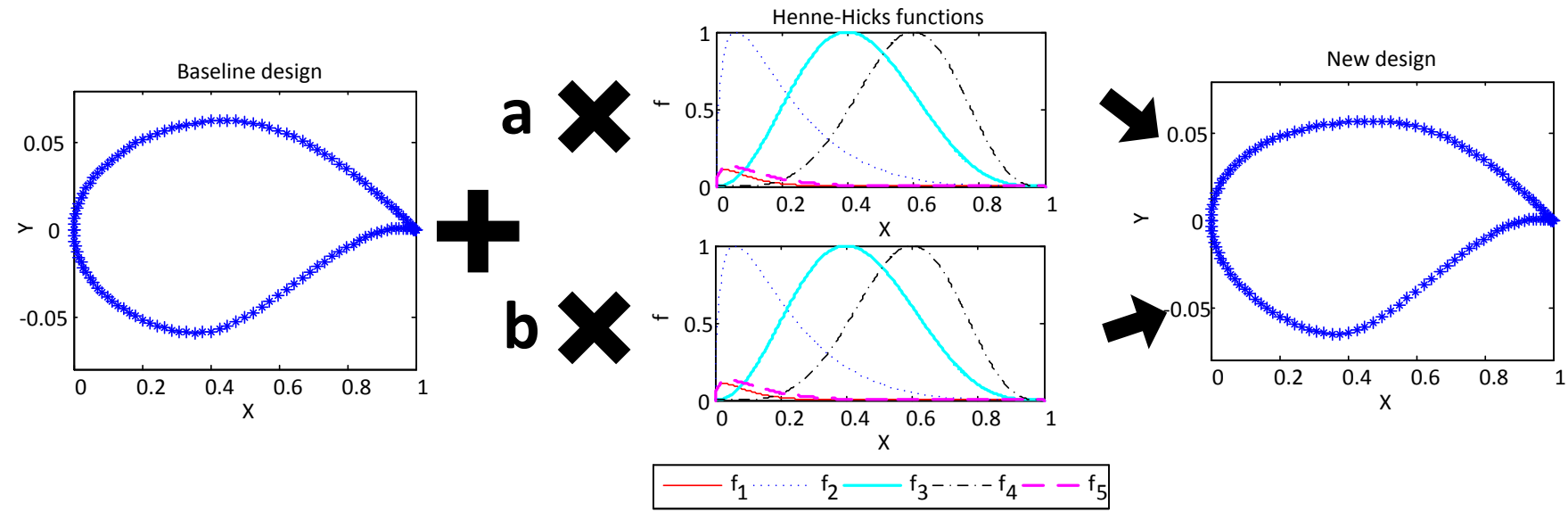

Fig. 2. Geometry parameterization using Henne-Hicks functions

1) Geometry Parameterization: The airfoil geometry is defined in a two-dimensional space with $x$ and $y$ coordinates, as shown in Fig. 2 where a baseline design is depicted. The geometry of the baseline design can be modified by using the Henne-Hicks functions [12] as follows.

$$
\begin{gathered}
f_{1}=\frac{x^{0.5}(1-x)}{x^{15 x}} \\
f_{2}=\sin \left(\pi x^{0.25}\right)^{3} \\
f_{3}=\sin \left(\pi x^{0.757}\right)^{3} \\
f_{4}=\sin \left(\pi x^{1.357}\right)^{3} \\
f_{5}=\frac{x^{0.5}(1-x)}{x^{10 x}}
\end{gathered}
$$

Fig. 2 illustrates how a new airfoil geometry can be generated by adding the weighted sum of the above five functions to the baseline design, which can be described by Equations (6) and (7):

$$
\begin{gathered}
y_{u}=y_{u}^{b}+\sum_{i=1}^{5} a_{i} f_{i}, \\
y_{l}=y_{l}^{b}+\sum_{i=1}^{5} b_{i} f_{i},
\end{gathered}
$$

where $y_{u}$ and $y_{l}$ are the upper and lower surfaces of the geometry, $y_{u}^{b}$ and $y_{l}^{b}$ are the upper and lower surfaces of the baseline design, and $a_{i}$ and $b_{i}$ are weights. Thus, ten parameters $\left(a_{1}, \ldots, a_{5}\right.$ and $\left.b_{1}, \ldots, b_{5}\right)$ determining the shape are the decision variables of the airfoil design optimization problem.

2) Objective Function: Multiple criteria can be used to evaluate the performance of a given airfoil if its full-scenario wave drag landscape $\left(C_{D w}\left(M, C_{L}\right)\right)$ is available. The drag divergence boundary $B_{D D}$ is defined as the boundary at which the drag begins to increase rapidly as $M$ keeps increasing. The location of $B_{D D}$ in the two-dimensional scenario space $\left(M \times C_{L}\right)$ is an effective evaluation criterion [9], which can be identified according to the partial derivatives of $C_{D w}\left(M, C_{L}\right)$ with respect to $M$, as shown in Equation (8).

$$
\frac{\partial C_{D w}}{\partial M}=0.1
$$

Fig. 3 is a full-scenario landscape $C_{D w}\left(M, C_{L}\right)$ for an example airfoil, where the drag divergence boundary is shown by a line in pink based on Equation (8).

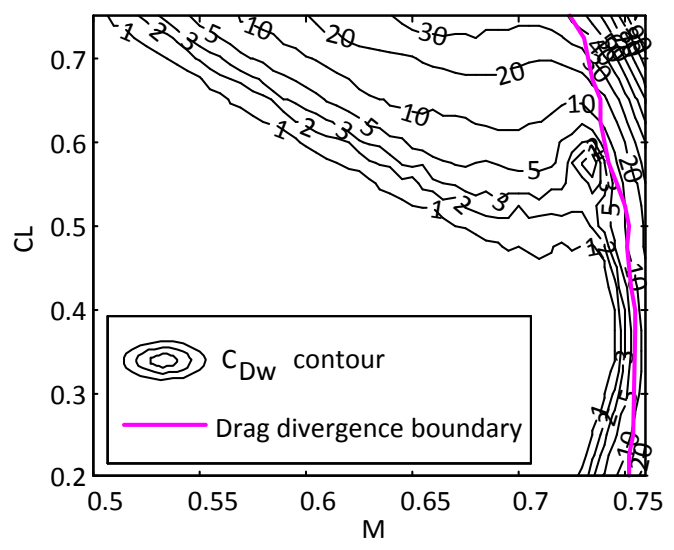

Fig. 3. Drag divergence boundary in the full scenario space.

Beyond the boundary $B_{D D}$, i.e., the scenario area to the right side of the drag divergence boundary (Fig. 3), the airfoil tends to operate at a higher speed (mach coefficient) requiring a larger amount of thrust (due to larger drag), which is undesired. Ideally, the drag divergence boundary $B_{D D}$ should be pushed to the extreme scenario $\left(M_{\max }, C_{L \max }\right)$, i.e., the area to the right of $B_{D D}$ in the scenario space should be minimized, resulting in an airfoil design that can efficiently operate at a high speed with small drag.

Thus, the location of the boundary $B_{D D}$ is able to quantitatively evaluate the full-scenario performance of airfoil. Therefore, in this study, the objective function for multiscenario optimization aims to minimize the area between the 
boundary and the extreme point $\left(M_{\max }, C_{L \max }\right)$. Let the scenario parameters $M$ and $C_{L}$ vary in $\left[M_{\min }, M_{\max }\right]$ and $\left[C_{L \text { min }}, C_{L \text { max }}\right]$, the objective function can be expressed as follows:

$$
\begin{aligned}
\min f_{B_{D D}}(\mathbf{a}, \mathbf{b}) & =S\left(B_{D D}(\mathbf{a}, \mathbf{b}), r\right) \\
\mathbf{a} & =\left[a_{1}, \ldots, a_{5}\right] \\
\mathbf{b} & =\left[b_{1}, \ldots, b_{5}\right] \\
r & =\left(M_{\max }, C_{L \max }\right),
\end{aligned}
$$

where $\mathbf{a}$ and $\mathbf{b}$ are the decision variables representing the airfoil geometry, $B_{D D}(\mathbf{a}, \mathbf{b})$ is the location of the drag divergence boundary of the airfoil, $r$ is the extreme scenario $\left(M_{\max }, C_{L \max }\right)$, and function $S$ is the area between the boundary and the extreme scenario. It is worth noting that function $S$ calculates a normalized area according to the ranges of $M$ and $C_{L}$, i.e., the area equals to 1 when $B_{D D}(\mathbf{a}, \mathbf{b})$ locates on $\left(M_{\min }, C_{L \min }\right)$.

\section{HiERARCHICAL SURRogATE-ASSISTED Multi-ScEnARIo Evolution StRATEGY}

\section{A. Overall Framework}

To perform multi-scenario airfoil design, the entire 2D scenario space is firstly discretized into $N_{M} \times N_{C_{L}}$ evenly distributed scenarios. Then, CFD simulations are performed for each of the scenarios to calculate the objective defined in Equation (9). Thus, $N_{M} \times N_{C_{L}}$ CFD simulations need to be conducted for each airfoil design, resulting in extremely high computational cost for multi-scenario optimization.

Since multi-scenario airfoil design optimization using CFD simulations is computationally extremely expensive, this work proposes a hierarchical surrogate modelling technique to approximate the full-scenario drag landscape, based on which the objective value of in Equation (9) is calculated to reduce the computational cost. The proposed algorithm employs a CMAES assisted by the hierarchical surrogate for multi-scenario airfoil optimization, which is termed HSA-MSES in short.

The overall diagram of HSA-MSES is provided in Fig. 4, from which we can see that HSA-MSES consists of three main components, i.e., the CMA-ES, the hierarchical surrogate for approximating the full-scenario drag landscape, and the calculation of the fitness.

\section{B. CMA-ES}

CMA-ES is well known for its fast convergence using a relatively small population size [11]. In CMA-ES, $\lambda$ offspring solutions are generated in each generation $g$ from an $n$ dimensional multivariate normal distribution $N\left(\mathbf{m}^{g}, \sigma^{\mathbf{g}^{2}} \mathbf{C}^{\mathbf{g}}\right)$, where $\mathbf{m}^{g}$ is the mean, $\sigma^{\mathbf{g}}$ is the standard deviation, and $\mathbf{C}^{\mathbf{g}}$ is the covariance matrix between decision variables. Then the best $\mu$ individuals are selected as the parents of next generation $(g+1)$ and the distribution will be updated accordingly as follows:

$$
\begin{aligned}
\mathbf{m}^{g+1} & =\sum_{i=1}^{u} w_{i} \mathbf{x}_{i} \\
w_{i} & =\frac{\ln (\mu+0.5)-\ln i}{\sum_{j=1}^{u} \ln (\mu+0.5)-\ln j}
\end{aligned}
$$

The standard deviation and the covariance matrix are updated as described below:

$$
\begin{aligned}
\mathbf{C}^{g+1} & =\left(1-\frac{2}{n^{2}}-\frac{0.3 \lambda}{n^{2}}\right) \mathbf{C}^{g}+\frac{2}{n^{2}} \mathbf{p}_{\mathbf{c}}{ }^{g+1}\left(\mathbf{p}_{\mathbf{c}}{ }^{g+1}\right)^{T}+ \\
& \frac{0.3 \lambda}{n^{2}} \sum_{i=1}^{u} w_{i} \frac{\mathbf{x}_{i}-\mathbf{m}^{g}}{\sigma^{g}}\left(\frac{\mathbf{x}_{i}-\mathbf{m}^{g}}{\sigma^{g}}\right)^{T} \\
\sigma^{g+1} & =\sigma^{g} \times \exp \left(\frac{4 / n}{1+\sqrt{0.3 \lambda / n}}\left(\frac{\left\|\mathbf{p}_{\sigma}{ }^{g+1}\right\|}{E\|N(\mathbf{0}, \mathbf{I})\|}-1\right)\right)
\end{aligned}
$$

where, $\mathbf{p}_{\mathbf{c}}{ }^{g+1}$ and $\mathbf{p}_{\sigma}{ }^{g+1}$ are known as evolution paths and can be adapted according to the following equations:

$$
\begin{aligned}
\mathbf{p}_{\mathbf{c}}{ }^{g+1} & =\left(1-\frac{4}{n}\right) \mathbf{p}_{\mathbf{c}}{ }^{g}+ \\
1_{\left\{\left\|p_{\sigma}{ }^{g}\right\|<1.5 \sqrt{n}\right\}} \sqrt{1-\left(1-\frac{4}{n}\right)^{2}} \sqrt{0.3 \lambda} \frac{\mathbf{m}^{g+1}-\mathbf{m}^{g}}{\sigma^{g}} & \\
\mathbf{p}_{\sigma}{ }^{g+1} & =\left(1-\frac{4}{n}\right) \mathbf{p}_{\sigma^{g}}+ \\
& \sqrt{1-\left(1-\frac{4}{n}\right)^{2}} \sqrt{0.3 \lambda}\left(\mathbf{C}^{g}\right)^{-0.5} \frac{\mathbf{m}^{g+1}-\mathbf{m}^{g}}{\sigma^{g}}
\end{aligned}
$$

where $1_{\left\{\left\|p_{\sigma} g\right\|<1.5 \sqrt{n}\right\}}$ is an indicator function that evaluates to 1 when $\left\|p_{\sigma}^{g}\right\|<1.5 \sqrt{n}$ or otherwise 0 .

\section{Hierarchical Surrogate Modeling and Infill Sampling Cri- terion}

Surrogates such as Kriging models (Gaussian processes), polynomial regression, and radial basis function networks have widely been employed to assist evolutionary algorithms (EAs) for solving expensive engineering optimization problems [13]. A central challenge in surrogate-assisted evolutionary algorithms (SAEAs) is to manage the surrogates properly so that the EA is able to find a near-optimum with limited computational budget [14].

In this work, the surroagte is supposed to approximate the full-scenario drag landscpe for fitness estimation The approximation of the full-scenario drag landscape for a given airfoil can be seen as a two-dimensional regression problem. In this work, we adopt a Kriging model [15] to approximate the drag scenario, which is trained using CFD simulation results collected from 22 scenarios. This is based on the recomendation in [16] suggesting that a minimum of $11 d$ samples should be generated for constructing Kriging models, where $d$ is the dimension of the function to be approximated.

Taking a closer look at the drag landscape as shown in Fig. 3 , we find that the drag equals 0 in most of the lower-left region of the drag landscape. Thus, additional cheap training data can be generated for scenarios in the zero drag region if the boundary between the zero drag and non-zero drag can be estimated using the 22 CFD simulations. To this end, we build a K-nearest neighbors (KNN) classifer [17] using the training data from 22 scenarios to predict whether a given scenario is a zero or non-zero drag scenario. Then, new training data will be generated for the zero drag scenarios. After that, a Kriging model is constructed. The drag divergence boundary $B_{D D}$ will then be detected according to the approximated drag landscape 


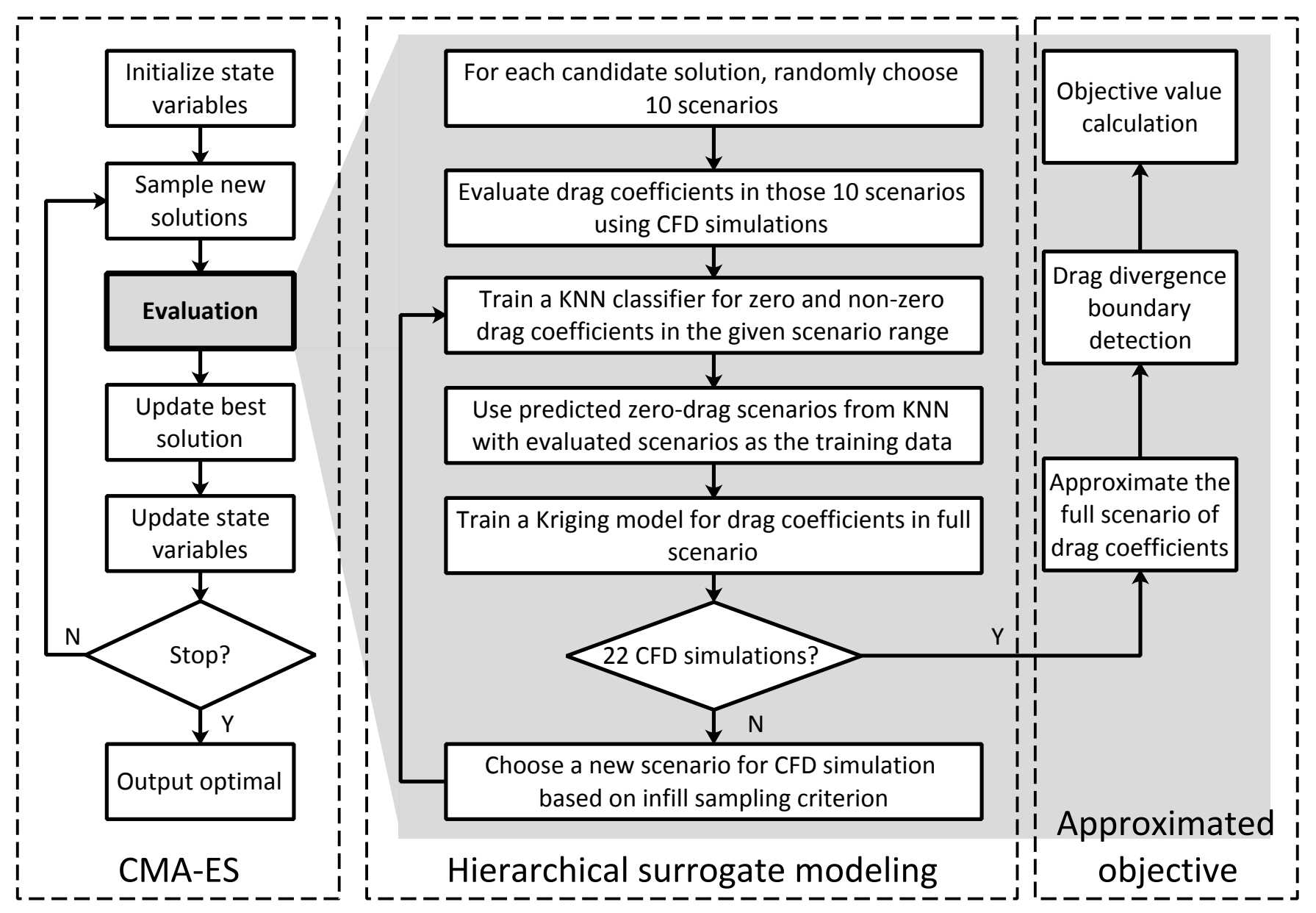

Fig. 4. The overall diagram of HSA-MSES.

$\left.\hat{C}_{D w}\left(M, C_{L}\right)\right)$, based on which the fitness of the candidate design can be calculated. The main steps for constructing the hierarchical surrogate are shown in Fig. 5, where $D$ denotes the training data collected from $N_{M} \times N_{C_{L}}$ scenarios and additional training data generated generated from the zero drag region are denoted by $D_{0}$. Finally, the Kriging model is trained using $D \cup D_{0}$ to approximate the full-scenario drag landscape of the given airfoil.

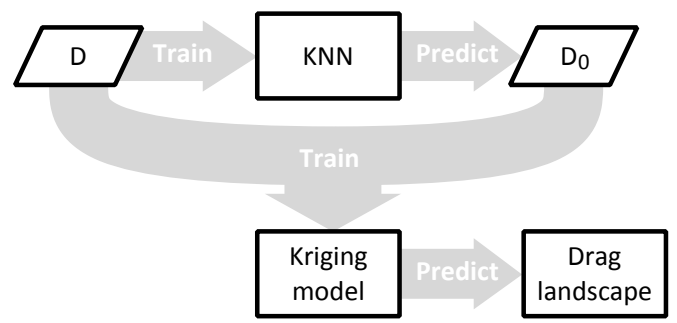

Fig. 5. Hierarchical surrogate modeling in HSA-MSES. A KNN classifier is trained based on $D$ (training data generated using CFD simulations) to identify the zero-drag scenarios $\left(D_{0}\right)$ among the $N_{M} \times N_{C_{L}}$ candidate scenarios. Then, the Kriging model is trained using $D \cup D_{0}$ to approximate the fullscenario drag landscape.
The 22 scenarios for performing CFD simulations are determined using two different criteria: 10 scenarios are randomly chosen from those $N_{M} \times N_{C_{L}}$ candidate scenarios and the rest 12 are selected according to a new infill sampling criterion aiming to improve the approximation accuracy around the drag divergence boundary.

The lower confidence bound (LCB) criterion [18] is an infill sampling criterion widely used for Kriging assisted optimization algorithms. However, LCB is not best suited for the drag landscape approximation, because we are mainly concerned about the approximation accuracy around the drag divergence boundary rather than the optimum (the minimal drag) of the drag landscape. Therefore, we modify the original LCB criterion as follows:

$$
\underset{\left(M, C_{L}\right)}{\arg \min } f_{l c b}=\left|\frac{\partial \hat{C}_{D w}\left(M, C_{L}\right)}{\partial M}-0.1\right|-w \sigma_{D w}\left(M, C_{L}\right),
$$

where $\hat{C}_{D w}\left(M, C_{L}\right)$ and $\sigma_{D w}\left(M, C_{L}\right)$ are the mean and standard deviation of the predicted drag coefficient, respectively. In Equation (15), the first term is to detect the boundary rather than the optimum, while the second term is the uncertainty of the Kriging model, and $w$ is a weigh to balance the two terms 
defined as follows.

$$
w=\frac{\alpha_{1}}{\alpha_{2}}
$$

where $\alpha_{1}$ and $\alpha_{2}$ are the mean $\left|\frac{\partial \hat{C}_{D w}\left(M, C_{L}\right)}{\partial M}-0.1\right|$ and $\sigma_{D w}\left(M, C_{L}\right)$ of $N_{M} \times N_{C_{L}}$ candidate scenarios.

After performing CFD simulations for the 10 randomly chosen scenarios, 12 scenarios are successively selected according to the $f_{l c b}$ value of $N_{M} \times N_{C_{L}}$ candidate scenarios. The successive selection using the infill criterion consists of three steps. Firstly, the scenario with the minimal $f_{l c b}$ value among $N_{M} \times N_{C_{L}}$ candidate scenarios is selected. Then, CFD simulations for the selected scenario is conducted and the resulting data is added to $D$. Finally, both $\mathrm{KNN}$ and Kriging model are updated. When CFD simulations for all 22 scenarios have been performed, the objective value of a candidate airfoil design is estimated using the kriging model approximating the drag landscape.

Fig. 6 illustrates the process of the hierarchical surrogate modeling. In sub-figure (A), 10 scenarios are randomly chosen as the initial training data and 12 scenarios are sequentially selected using the infill sampled criterion. The drag coefficient for these 22 scenarios are calculated using CFD simulations. Then, in sub-figure (B), a KNN classifier is trained from the data from from the 22 scenarios to identify the zero drag region. Finally, in sub-figure (C), the Kriging model is constructed based on $22 \mathrm{CFD}$ simulation data and additional data predicted in the zero drag region.

\section{Drag Divergence Boundary Detection and Objective Func- tion Calculation}

Given the approximated full-scenario drag landscape $\hat{C}_{D w}\left(M, C_{L}\right)$, we can detect a number of scenarios satisfying Equation (8). A linear function can then be identified to describe the drag divergence doundary $B_{D D}$ using the leastsquares method [19]:

$$
C_{L}=k M+d,
$$

where $k$ and $d$ are two parameters to be estimated. Note, however, that $B_{D D}$ is not exactly linear and therefore the linear model in Equation (8) will result in an approximation error $E$ (measured by mean absolute error). Thus, the divergence drag boundary taking the approximation error into account can be rewritten as follows:

$$
C_{L}=k M+d-E
$$

Fig. 7 is an example illustrating the process of objective calculation in the proposed algorithm. Firstly, the scenarios on the boundary $B_{D D}$ (denoted by stars in Fig. 7) are detected based on the predicted drag landscape. Then, a linear function for $B_{D D}$ is built using the detected scenarios (denoted by the solid line). The drag divergence boundary $B_{D D}$ compensated by the approximation error in Equation (18) is denoted by the dotted line. Finally, the objective value of the candidate design can be obtained by calculating the area between the compensated drag divergence boundary $B_{D D}$ and the extreme scenario on the top right of the scenario space.

\section{EXPERIMENTAL RESULTS}

In this section, we empirically examine the performance of the proposed HSA-MSES on using RAE2822 airfoil design as an example. As shown in Section II-B1, the shape an airfoil can be represented by $(\mathbf{a}, \mathbf{b})$ and the search space is defined in $[-0.01,0.01]^{10}$. The 2D scenario space of RAE2822 airfoil is defined by $M \in[0.6,0.76] \times C_{L} \in[0.5,0.85]$ and divided into $35 \times 15$ even grid [9] for multi-scenario optimization. Therefore, an exact full-scenario evaluation of a single airfoil needs 525 CFD simulations.

For the KNN classifier in the proposed algorithm, we set the number of neighbors $K$ to 2 and use the Mahalanobis distance as the distance metric. For the Kriging model, we use a simple Kriging model [20] with a Gaussian kernel, which is implemented by DACE toolbox [21] using the Hooke \& Jeeves method [22] to optimize its hyperparameters.

As discussed before, 22 CFD simulations needs to be performed for a single shape in HSA-MSES. Considering the high computational cost, we use a relatively fast CFD simulation tool, VGK [23], [24] for evaluating the drag, each simulation taking about one minute. Finally, the parent and offspring population sizes of CMA-ES are set to be 6 and 11, respectively, and the maximum number of generations is set to 50 .

\section{A. Approximation Accuracy of Surrogate Models}

To evaluate the accuracy of the hierarchical surrogate model in HSA-MSES, $35 \times 15$ CFD simulations are performed on the baseline design to obtain its full-scenario drag landscape. The drag divergence boundary is then detected as the ground truth. We first compare approximation performance of the proposed hierarchical surrogate model with a plain Kriging model trained using $22 \mathrm{CFD}$ data only. The approximation error is measured by the average distance from the approximated drag divergence boundary to the true boundary. the results are repeated independently for 20 times.

Our results show that the hierarchical surrogate model achieves an approximation error of $0.0028 \pm 0.0005$ while the Kriging model achieves an approximation error of $0.0100 \pm 0.0118$. These results demonstrate that the additional data generated from the KNN classifier improves the accuracy of the Kriging model in approximating the drag divergence boundary.

\section{B. Application to RAE2822 Airfoil Design}

Because of the extremely high computational cost, we are able to run the proposed algorithm on the RAE2822 airfoil design problem for only once. The convergence profile of HSA-MSES is shown in Fig. 8, from which we can see that HSA-MSES is able to converge very quickly within less than 20 generations.

Figs. 9 and 10 show the full-scenario drag landscape of the baseline design and that of the optimal design obtained by HSA-MEES based on $35 \times 15$ CFD simulations, where each full-scenario evaluation takes about 40 minutes. Comparing the drag divergence boundaries of the baseline design and the 

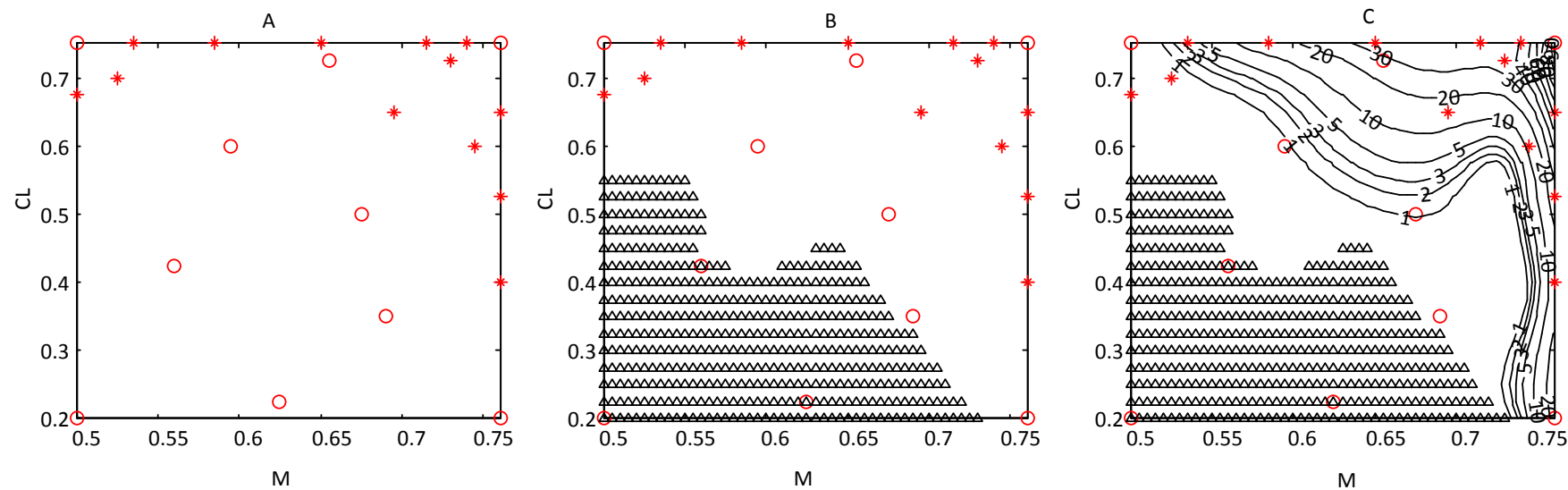

Randomly initialized scenarios $\Delta$ Zero-drag scenarios predicted by KNN

* Infill sampled scenarios (2) $C_{D w}$ contour predicted by Kriging model

Fig. 6. Illustration of hierarchical surrogate for approximating the drag landscape using a limited number of CFD simulations. (A) 10 scenarios are randomly chosen and 12 are selected sequentially using the infill criterion. (B) A KNN classifier is trained using the CFD simulation results of the 22 scenarios to identify the zero drag region. (C) The Kriging model is built based on the 22 CFD data and additional data generated from the KNN model.

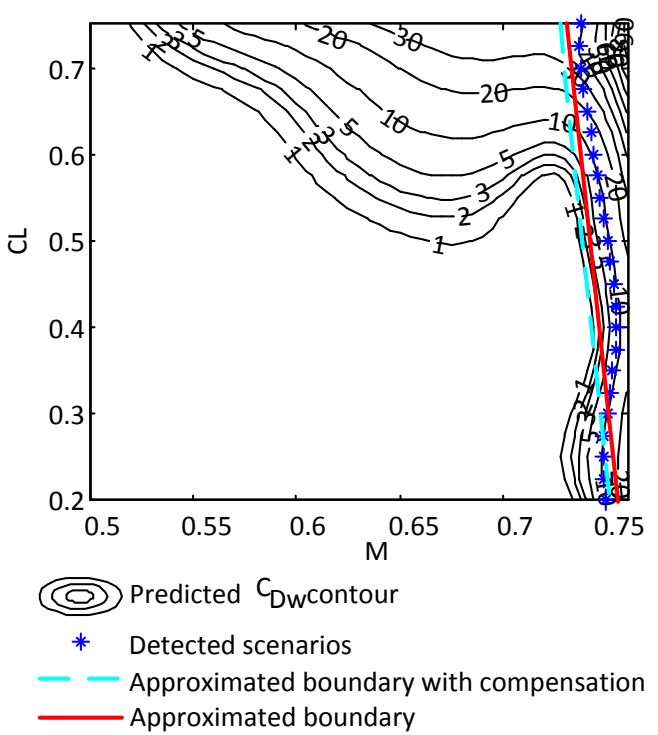

Fig. 7. Illustration of drag divergence boundary detection and objective calculation.

obtained design, we can clearly see that the boundary of the optimal design has moved towards the extreme scenario and the objective value is reduced from 0.248873 to 0.032607 , indicating a significantly improved full-scenario performance of the airfoil.

The optimization run of RAE2822 airfoil using HSA-MSES consumes 13,200 CFD simulations and a total amount of 14.5 hours, which allows for approximately 25 CFD simulation based full-scenario evaluations. In other words, only two generations of full-scenario optimization using CMA-ES can be run. It is conceivable that CMA-ES will not be able improve the performance a lot within only two generations.

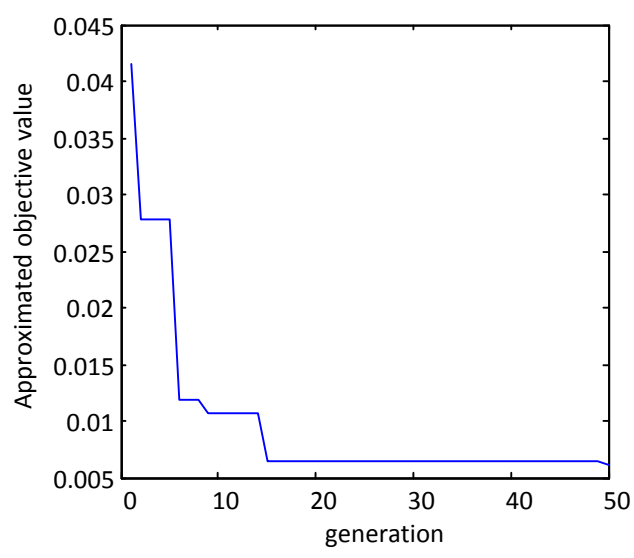

Fig. 8. Convergence profile of HSA-MSES on the RAE2822 airfoil design problem.

\section{Conclusion}

This paper addresses multi-scenario airfoil design optimization, which is challenging due to extremely high computational cost. To solve the problem, we first give a problem formulation for multi-scenario airfoil optimization that minimizes the area between the drag divergence boundary and extreme point on the drag landscape. To reduce the needed number of expensive CFD simulations as much as possible, a hierarchical surrogate consisting of a KNN and a Kriging model is proposed to assist the covariance matrix adaptation evolution strategy. Our experimental results on the RAE2822 airfoil design demonstrate that the proposed algorithm is able to find an optimal design having much improved aerodynamic performance compared to the baseline design using limited amount of computational budget.

Our future work will consider multi-scenario airfoil optimization taking both drag and lift into account. Robustness 


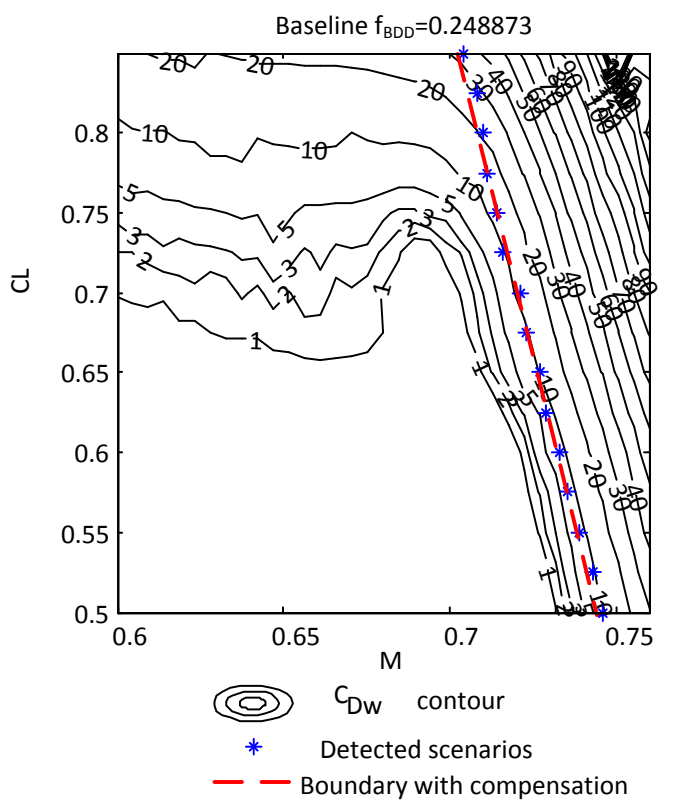

Fig. 9. Exact full-scenario drag landscape of the baseline design.

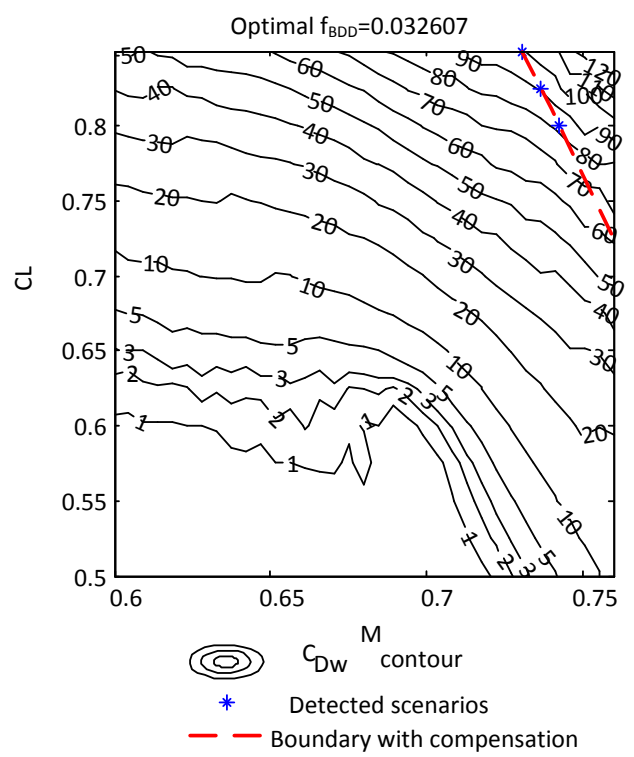

Fig. 10. Exact full-scenario drag landscape of the optimal design obtained by HSA-MSES.

in multi-scenario design will also be considered, which is even more challenging. Thus, new surrogate management techniques are highly in demand. Finally, it is of interest to extend the proposed method to full-/multi-scenario optimization of other engineering design problems such as design of turbine engines.

\section{REFERENCES}

[1] H.-G. Beyer and B. Sendhoff, "Robust optimization-a comprehensive survey," Computer Methods in Applied Mechanics and Engineering, vol. 196, no. 33, pp. 3190-3218, 2007.
[2] K. Deb, L. Zhu, and S. Kulkarni, "Handling multiple scenarios in evolutionary multi-objective numerical optimization," IEEE Transactions on Evolutionary Computation, vol. PP, no. 99, pp. 1-1, 2017, doi=10.1109/TEVC.2017.2776921.

[3] G. Palermo, C. Silvano, and V. Zaccaria, "Robust optimization of soc architectures: A multi-scenario approach," in Embedded Systems for Real-Time Multimedia, 2008. ESTImedia 2008. IEEE/ACM/IFIP Workshop on. IEEE, 2008, pp. 7-12.

[4] K. Deb, L. Zhu, and S. Kulkarni, "Multi-scenario, multi-objective optimization using evolutionary algorithms: Initial results," in IEEE Congress on Evolutionary Computation (CEC). IEEE, 2015, pp. 18771884.

[5] C.-S. Wang, B. Yu, J. Xiao, and L. Guo, "Multi-scenario, multiobjective optimization of grid-parallel microgrid," in 4th International Conference on Electric Utility Deregulation and Restructuring and Power Technologies (DRPT). IEEE, 2011, pp. 1638-1646.

[6] M. M. Wiecek, V. Singh, and V. Blouin, "Multi-scenario multi-criteria optimization in engineering design," Tech. Rep., 2007.

[7] L. Zhu, K. Deb, and S. Kulkarni, "Multi-scenario optimization using multi-criterion methods: A case study on byzantine agreement problem," in Evolutionary Computation (CEC), 2014 IEEE Congress on. IEEE, 2014, pp. 2601-2608.

[8] B. Li, J. Li, K. Tang, and X. Yao, "Many-objective evolutionary algorithms: A survey," ACM Computing Surveys (CSUR), vol. 48, no. 1 pp. $1-35,2015$.

[9] J. J. Doherty, "Transonic airfoil study using sonic plateau, optimization and off-design performance maps," in 35th AIAA Applied Aerodynamics Conference, 2017, p. 3056.

[10] Y. Jin, "Surrogate-assisted evolutionary computation: Recent advances and future challenges," Swarm and Evolutionary Computation, vol. 1, no. 2, pp. 61-70, 2011.

[11] N. Hansen and A. Ostermeier, "Completely derandomized selfadaptation in evolution strategies," Evolutionary Computation, vol. 9, no. 2, pp. 159-195, 2001.

[12] R. M. Hicks and P. A. Henne, "Wing design by numerical optimization," Journal of Aircraft, vol. 15, no. 7, pp. 407-412, 1978.

[13] H. Wang, Y. Jin, and J. Doherty, "Committee-based active learning for surrogate-assisted particle swarm optimization of expensive problems," IEEE Transactions on Cybernetics, vol. 47, no. 9, pp. 2664-2677, 2017.

[14] Y. Jin, "A comprehensive survey of fitness approximation in evolutionary computation," Soft Computing, vol. 9, no. 1, pp. 3-12, 2005.

[15] C. K. Williams, "Prediction with gaussian processes: From linear regression to linear prediction and beyond," Learning and Inference in Graphical Models, vol. 89, pp. 599-621, 1998.

[16] D. R. Jones, M. Schonlau, and W. J. Welch, "Efficient global optimization of expensive black-box functions," Journal of Global optimization, vol. 13, no. 4, pp. 455-492, 1998.

[17] N. S. Altman, "An introduction to kernel and nearest-neighbor nonparametric regression," The American Statistician, vol. 46, no. 3, pp. $175-185,1992$

[18] J. Dennis and V. Torczon, Managing approximation models in optimization. Philadelphia, PA: SIAM, 1997.

[19] S. J. Leon, Linear algebra with applications. Macmillan New York, 1980.

[20] R. A. Olea, "Geostatistics for engineers and earth scientists," Technometrics, vol. 42, no. 4, pp. 444-445, 2000.

[21] S. Lophaven, H. Nielsen, and J. Sondergaard, "Dace: A matlab kriging toolbox. 2002," Technical University of Denmark, Tech. Rep., 2002.

[22] J. S. Kowalik and M. R. Osborne, Methods for unconstrained optimization problems. North-Holland, 1968.

[23] P. Ashill, R. Wood, and D. Weeks, "An improved, semi-inverse version of the viscous garabedian and korn method (VGK)," RAE TR, vol. 87002, 1987.

[24] M. Freestone, "VGK method for two-dimensional aerofoil sections part 1: principles and results," ESDU 96028, Tech. Rep., 2004. 